\title{
ON BINOMIAL COEFFICIENT RESIDUES
}

\author{
J. B. ROBERTS
}

The number of binomial coefficients $\left(\begin{array}{l}u \\ v\end{array}\right), 0 \leqslant v \leqslant u<n$, which are congruent to $j, 0 \leqslant j \leqslant p-1$, modulo the prime number $p$ is denoted by $\theta_{j}(n)$. In this paper we give systems of simultaneous linear difference equations with constant coefficients whose solutions would yield the quantities $\theta_{j}(n)$ explicitly. In this direction we compute $\theta_{j}(n)$ in all cases for $p=2$ and $\theta_{j}\left(p^{k}\right), k \geqslant 0$, in all cases for $p=3$ or 5 . The complete explicit determination of $\theta_{j}(n)$ for arbitrary $n$ is quite tedious for $p>2$.

We also include various special results in the case $p=2$ and prove that every prime divides "most" binomial coefficients in the sense that

$$
\lim _{\rightarrow \infty} \theta(n) / \theta_{0}(n)=0
$$

where

$$
\theta(n)=\sum_{j=1}^{p-1} \theta_{j}(n)
$$

1. Definitions. If $c, a, s, k$ are constants satisfying $0 \leqslant a \leqslant c \leqslant p-1$, $1 \leqslant s \leqslant p^{k}, \mathrm{k}>0$, then the collection of all $\left(\begin{array}{l}u \\ v\end{array}\right)$ satisfying

$$
c p^{k} \leqslant u<c p^{k}+s, a p^{k} \leqslant v \leqslant u+(a-c) p^{k},
$$

will be denoted by $(c, s, a)_{k}$. When we write $(c, s, a)_{k}$ we will assume that $c, a, s, k$ satisfy the specified conditions unless stated explicitly to the contrary. For instance if we write $(0, s, a)_{k}$ this implies $0 \leqslant a \leqslant p-1,1 \leqslant s \leqslant p^{k}$, $k>0$. Any collection $(c, s, a)_{k}$ will be called a $k$-triangle.

The $k$-triangle $(c, s, a)_{k}$ can be put into 1-1 correspondence with the $k$ triangle $(0, s, 0)_{k}$ by the mapping

$$
\left(\begin{array}{l}
u \\
v
\end{array}\right) \leftrightarrow\left(\begin{array}{l}
u-c p^{k} \\
v-a p^{k}
\end{array}\right)
$$

Hence any two $k$-triangles can be put into $1-1$ correspondence. Corresponding elements will be called homologous.

If $K_{1}$ and $K_{2}$ are two $k$-triangles and $\alpha$ is an integer such that $k_{1} \equiv \alpha k_{2}$ $(\bmod p)$ whenever $k_{1} \in K_{1}$ and $k_{2} \in K_{2}$ are homologous we will write $K_{1} \equiv$ $\alpha K_{2}(\bmod p)$.

Received October 2, 1956 
2. A lemma of Lucas and applications. Our first lemma is a result of Lucas (1, p. 271). A simple proof may be found in Glaisher (2). We use $p$ for a prime throughout.

Lemma 1. If in the scale of radix $p$,

$$
\begin{aligned}
& m=b_{0}+b_{1} p+\ldots+b_{k} p^{k} \\
& n=a_{0}+a_{1} p+\ldots+a_{k} p^{k},
\end{aligned}
$$

then

$$
\left(\begin{array}{l}
n \\
m
\end{array}\right) \equiv\left(\begin{array}{l}
a_{0} \\
b_{0}
\end{array}\right) \cdots\left(\begin{array}{c}
a_{k} \\
b_{k}
\end{array}\right)
$$

(The quantity $\left(\begin{array}{l}r \\ s\end{array}\right)=0$ when $s>r$.)

Before making use of this lemma we observe that by repeated use of the identity

$$
\left(\begin{array}{l}
a \\
b
\end{array}\right)+\left(\begin{array}{c}
a \\
b+1
\end{array}\right)=\left(\begin{array}{l}
a+1 \\
b+1
\end{array}\right)
$$

and the almost obvious fact that

$$
\left(\begin{array}{c}
p^{k} \\
m
\end{array}\right) \equiv 0(\bmod p) \quad\left(1 \leqslant m \leqslant p^{k}-1, \quad k>0\right)
$$

we are able to prove

Lemma 2. If $n-p^{k}+1 \leqslant m<p^{k} \leqslant n<2 p^{k}-1, k>0$, then

$$
\left(\begin{array}{l}
n \\
m
\end{array}\right) \equiv 0
$$

$(\bmod p)$

We come now to our first application of Lemma 1.

Lemma 3. If $0 \leqslant v \leqslant u, c p^{k} \leqslant u<(c+1) p^{k}, \quad 1 \leqslant c \leqslant p-1$ and if $\left(\begin{array}{l}u \\ v\end{array}\right)$ is in none of the k-triangles $\left(c, p^{k}, a\right)_{k}, 0 \leqslant a \leqslant c$, then

$$
\left(\begin{array}{l}
u \\
v
\end{array}\right) \equiv 0
$$

Proof. Since $\left(\begin{array}{l}u \\ v\end{array}\right)$ is not in $\left(c, p^{k}, a\right)_{k}$ for each $a, 0 \leqslant a \leqslant c, v$ must satisfy for some $a, 0 \leqslant a \leqslant c-1$, the inequality

$$
u+1+(a-c) p^{k} \leqslant v \leqslant(a+1) p^{k}-1 .
$$

Since for each $a, 0 \leqslant a \leqslant c-1$, this inequality is impossible when $u=(c+1) p^{k}-1$ we can restrict attention to $u<(c+1) p^{k}-1$. Now

$$
\begin{aligned}
& u=a_{0}+a_{1} p+\ldots+a_{k-1} p^{k-1}+c p^{k} \\
& v=b_{0}+b_{1} p+\ldots+b_{k-1} p^{k-1}+a p^{k}
\end{aligned}
$$


and therefore by Lemma 1 ,

$$
\left(\begin{array}{l}
u \\
v
\end{array}\right) \equiv\left(\begin{array}{l}
a_{0} \\
b_{0}
\end{array}\right) \ldots\left(\begin{array}{l}
a_{k-1} \\
b_{k-1}
\end{array}\right)\left(\begin{array}{l}
c \\
a
\end{array}\right) \equiv\left(\begin{array}{l}
u-(c-1) p^{k} \\
v-a p^{k}
\end{array}\right) \quad(\bmod p) .
$$

But since

$$
\begin{aligned}
\left(u-(c-1) p^{k}\right)-p^{k}+1 & =u+1-c p^{k} \leqslant v-a p^{k} \leqslant p^{k}-1 \\
& <p^{k} \leqslant u-(c-1) p^{k}<2 p^{k}-1,
\end{aligned}
$$

we know by Lemma 3 that

$$
\left(\begin{array}{l}
u-(c-1) p^{k} \\
v-a p^{k}
\end{array}\right) \equiv 0
$$

This completes the proof.

By this lemma we see that when $u \neq(c+1) p^{k}-1$ there is always a $v$, $0 \leqslant v \leqslant u$, such that $\left(\begin{array}{l}u \\ v\end{array}\right)$ is divisible by $p$. It is interesting to note that for each $u$ of the form $(c+1) p^{k}-1,\left(\begin{array}{l}u \\ v\end{array}\right)$ is non-divisible by $p$ for $0 \leqslant v \leqslant u$. Thus we state the

Corollary. No $\left(\begin{array}{l}u \\ v\end{array}\right), 0 \leqslant v \leqslant u$, is divisible by $p$ if and only if $u$ is of the form $(c+1) p^{k}-1$ where $0 \leqslant c \leqslant p-1$.

Proof. The necessity is by the lemma. For the sufficiency we have

$$
\begin{gathered}
(c+1) p^{k}-1=(p-1)+(p-1) p+\ldots+(p-1) p^{k-1}+c p^{k} \\
v=b_{0}+b_{1} p+\ldots+b_{k-1} p^{k-1}+b_{k} p^{k}
\end{gathered}
$$

where $b_{i} \leqslant p-1,1 \leqslant i \leqslant k-1$ and $b_{k} \leqslant c$. Hence

$$
\left(\begin{array}{c}
(c+1) p^{k}-1 \\
v
\end{array}\right) \equiv\left(\begin{array}{c}
p-1 \\
b_{0}
\end{array}\right) \ldots\left(\begin{array}{c}
p-1 \\
b_{k-1}
\end{array}\right)\left(\begin{array}{c}
c \\
b_{k}
\end{array}\right) \quad(\bmod p)
$$

by Lemma 1 . But this right-hand side is not congruent to 0 modulo $p$. This completes the proof.

Another important application of Lemma 1 is the following

LEMMA 4.

$$
(c, s, a)_{k} \equiv\left(\begin{array}{c}
c \\
a
\end{array}\right)(0, s, 0)_{k} \quad(\bmod \mathrm{p})
$$

Proof. Let $\left(\begin{array}{l}u \\ v\end{array}\right)$ be in $(c, s, a)_{k}$. Then $c p^{k} \leqslant u<c p^{k}+s, a p^{k} \leqslant v \leqslant u$ $+(a-c) p^{k}$ and we can write in radix $p$, 


$$
\begin{aligned}
& u=a_{0}+a_{1} p+\ldots+a_{k-1} p^{k-1}+c p^{k} \\
& v=b_{0}+b_{1} p+\ldots+b_{k-1} p^{k-1}+a p^{k} .
\end{aligned}
$$

Hence by Lemma 1,

$$
\left(\begin{array}{l}
u \\
v
\end{array}\right) \equiv\left(\begin{array}{l}
u-c p^{k} \\
v-a p^{k}
\end{array}\right)\left(\begin{array}{l}
c \\
a
\end{array}\right)
$$

Since $\left(\begin{array}{c}u-c p^{k} \\ v-a p^{k}\end{array}\right)$ runs over $(0, s, 0)_{k}$ as $\left(\begin{array}{l}u \\ v\end{array}\right)$ runs over $(c, s, a)_{k}$ the proof is complete.

COROLlary. The number of numbers in $(c, s, a)_{k}$ which are congruent to $j(\bmod p), 1 \leqslant j \leqslant p-1, i s$

$$
\theta_{j_{a}}(s)
$$

where $j_{a}$ is that number satisfying

$$
1 \leqslant j_{a} \leqslant p-1, j_{a}\left(\begin{array}{c}
c \\
a
\end{array}\right) \equiv j
$$

Proof. By the lemma a number in $(c, s, a)_{k}$ is congruent to $j$ modulo $p$ if and only if $\left(\begin{array}{l}c \\ a\end{array}\right)$ times its homologous element in $(0, s, 0)_{k}$ is congruent to $j$ modulo $p$. Since

$$
j_{a}\left(\begin{array}{c}
c \\
a
\end{array}\right) \equiv j(\bmod \mathrm{p})
$$

the number of possibilities is the number of $j_{a}$ in $(0, s, 0)_{k}$ and this is just

$$
\theta_{j_{n}}(s) .
$$

3. The main recursion relation. Utilizing Lemma 3 we see that for $0 \leqslant c \leqslant p-1,1 \leqslant s \leqslant p^{k}$ all of those $\left(\begin{array}{l}u \\ v\end{array}\right), 0 \leqslant v \leqslant u, c p^{k} \leqslant u<c p^{k}+s$, which are not congruent to zero modulo $p$ are in one of the $c+1 k$-triangles $(c, s, a)_{k}, 0 \leqslant a \leqslant c$. Therefore $\theta_{j}\left(c p^{k}+s\right)-\theta_{j}\left(c p^{k}\right)$ is just the number of elements congruent to $j$ modulo $p$ contained in these $k$-triangles. By Lemma 4 this number is

$$
\sum_{a=0}^{c} \theta_{j_{a}}(s)
$$

Defining $e_{q j}(c), 1 \leqslant q \leqslant p-1$, to be the number of $j_{a}, 0 \leqslant a \leqslant c$, which are equal to $q$, the above sum becomes

$$
\sum_{q=1}^{p-1} e_{q j}(c) \theta_{q}(s)
$$

But $e_{q j}(c)$ is just the number of solutions of the congruence 


$$
\left(\begin{array}{c}
c \\
x
\end{array}\right) q \equiv j
$$

$(\bmod p)$,

which number is, by definition,

$$
\theta_{j \bar{q}}(c+1)-\theta_{j \bar{q}}(c)
$$

where $\bar{q}$ is the reciprocal of $q$ modulo $p$. Hence we have the following theorem setting forth our main recursion relation.

Theorem 1. If $0 \leqslant c \leqslant p-1,1 \leqslant s \leqslant p^{k}, k>0, q \bar{q} \equiv 1(\bmod p)$ then

$$
\theta_{j}\left(c p^{k}+s\right)=\theta_{j}\left(c p^{k}\right)+\sum_{q=1}^{p-1}\left(\theta_{j \bar{q}}(c+1)-\theta_{j \bar{q}}(c)\right) \theta_{q}(s) .
$$

Remembering the definition of $\theta(n)$ we have under the hypotheses of the theorem the following

Corollary 1. $\theta\left(c p^{k}+s\right)=\theta\left(c p^{k}\right)+(c+1) \theta(\mathrm{s})$.

Proof. For each $q, 1 \leqslant q \leqslant p-1$, the residues modulo $p$ of the numbers $\bar{q}, 2 \bar{q}, \ldots,(p-1) \bar{q}$ are the numbers $1,2, \ldots, p-1$ in some order. Using this fact and the theorem we obtain

$$
\begin{aligned}
\theta\left(c p^{k}+s\right) & =\sum_{j=1}^{p-1} \theta_{j}\left(c p^{k}+s\right) \\
& =\sum_{j=1}^{p-1} \theta_{j}\left(c p^{k}\right)+\sum_{q=1}^{p-1} \sum_{j=1}^{p-1}\left(\theta_{j \bar{q}}(c+1)-\theta_{j \bar{q}}(a)\right) \theta_{q}(s) \\
& =\theta\left(c p^{k}\right)+(\theta(c+1)-\theta(c)) \theta(s) .
\end{aligned}
$$

Since $\theta(c+1)-\theta(c)=c+1$, because $c$ is smaller than $p$, the proof is complete.

COROLlary 2. If $0 \leqslant c \leqslant p, k \geqslant 0$ then

$$
\begin{aligned}
& \theta_{j}\left(c p^{k}\right)=\sum_{q=1}^{p-1} \theta_{j \bar{q}}(c) \theta_{q}\left(p^{k}\right) ; \\
& \theta\left(c p^{k}\right)=\frac{1}{2} c(c+1) \theta\left(p^{k}\right) .
\end{aligned}
$$

Proof. (a) This is true for $c=0$ or $k=0$ so we suppose $c>0, k>0$. Now taking $s=p^{k}$ the theorem gives, for $1 \leqslant c \leqslant p$,

$$
\begin{aligned}
\theta_{j}\left(c p^{k}\right) & =\sum_{i=1}^{c}\left(\theta_{j}\left(i p^{k}\right)-\theta_{j}\left((i-1) p^{k}\right)\right) \\
& =\sum_{i=1}^{c} \sum_{q=1}^{p-1}\left(\theta_{j \bar{q}}(i)-\theta_{j \bar{q}}(i-1)\right) \theta_{q}\left(p^{k}\right) \\
& =\sum_{q=1}^{p-1} \sum_{i=1}^{c}\left(\theta_{j \bar{q}}(i)-\theta_{j \bar{q}}(i-1)\right) \theta_{q}\left(p^{k}\right) \\
& =\sum_{q=1}^{p-1} \theta_{j \bar{q}}(c) \theta_{q}\left(p^{k}\right) .
\end{aligned}
$$




$$
\begin{aligned}
\theta\left(c p^{k}\right) & =\sum_{j=1}^{p-1} \theta_{j}\left(c p^{k}\right) \\
& =\sum_{q=1}^{p-1}\left(\sum_{j=1}^{p-1} \theta_{j \bar{q}}(c)\right) \theta_{q}\left(p^{k}\right) \\
& =\theta(c) \theta\left(p^{k}\right)=\frac{1}{2} c(c+1) \theta\left(p^{k}\right) .
\end{aligned}
$$

Corollary 3. (a) If $k>0,1 \leqslant j \leqslant p-1$ then

$$
\theta_{j}\left(p^{k}\right)=\sum_{q=1}^{p-1} \theta_{j \bar{q}}(p) \theta_{q}\left(p^{k-1}\right) ;
$$

(b) If $k \geqslant 0$ then

$$
\theta\left(p^{k}\right)=\left(\frac{1}{2} p(p+1)\right)^{k}
$$

Proof. (a) Taking $c=p$ in Cor. 2 (a) gives

$$
\theta_{j}\left(p^{k+1}\right)=\sum_{q=1}^{p-1} \theta_{j \bar{q}}(p) \theta_{q}\left(p^{k}\right), k \geqslant 0
$$

and this is equivalent with (a).

(b) This is obvious for $k=0$. If true up to some $k \geqslant 0$ then by Cor. 2 (b),

$$
\theta\left(p^{k+1}\right)=\frac{1}{2} p(p+1) \theta\left(p^{k}\right)=\left(\frac{1}{2} p(p+1)\right)^{k+1} .
$$

This completes the proof.

By repeated application of these corollaries we are able to give an explicit expression for $\theta(n)$. This we do in the next corollary.

Corollary 4. If $n=a_{0}+a_{1} p+\ldots+a_{k} p^{k}, 0 \leqslant a_{i} \leqslant p-1$ then

$$
\theta(n)=\frac{1}{2} \sum_{i=0}^{k} a_{i}\left(\left(a_{i}+1\right) \ldots\left(a_{k}+1\right)\right)\left(\frac{1}{2} p(p+1)\right)^{i} .
$$

Theorem 1 and its corollaries determine the $\theta_{j}(n), 1 \leqslant j \leqslant n$, as solutions of a system of linear difference equations with constant coefficients. The quantity

$$
\theta_{0}(n)=\frac{1}{2} n(n+1)-\theta(n) .
$$

In general the calculations needed to compute explicitly the $\theta_{j}(n)$ are prohibitive. However we perform some calculations in this direction in the next section.

4. $\theta_{j}\left(p^{k}\right)$ for $p=3,5$. The simplest case to deal with is $p=2$. In this case we can compute $\theta_{j}(n)$ for arbitrary $n$. The details will be given in the next section where some other aspects of our results for $p=2$ are discussed.

When $p=3$, since $(j \bar{q}) q \equiv j(\bmod 3)$, we have

$$
1 \overline{1} \equiv 2 \overline{2} \equiv 1,1 \overline{2} \equiv 2 \overline{1} \equiv 2
$$

$(\bmod 3)$ 
By direct examination we find $\theta_{1}(3)=5, \theta_{2}(3)=1$. We now obtain from Cor. 3(a) of Theorem 1 the following pair of simultaneous difference equations

$$
\begin{aligned}
& \theta_{1}\left(3^{k}\right)-5 \theta_{1}\left(3^{k-1}\right)-\theta_{2}\left(3^{k-1}\right)=0, \\
& \theta_{2}\left(3^{k}\right)-\theta_{1}\left(3^{k-1}\right)-5 \theta_{2}\left(3^{k-1}\right)=0 .
\end{aligned}
$$

Solving these equations using the empirical initial conditions

$$
\theta_{1}(1)=\theta_{2}(3)=1, \theta_{1}(3)=5, \theta_{2}(1)=0
$$

we obtain

$$
\theta_{1}\left(3^{k}\right)=\frac{1}{2}\left(6^{k}+4^{k}\right), \theta_{2}\left(3^{k}\right)=\frac{1}{2}\left(6^{k}-4^{k}\right) .
$$

From these it follows that

$$
\theta_{0}\left(3^{k}\right)=\frac{1}{2} 3^{k}\left(3^{k}+1\right)-6^{k} .
$$

In a similar way with $p=5$ we find a system of four linear difference equations in four unknowns. Using the suitable initial conditions we obtain:

$$
\begin{aligned}
& \theta_{1}\left(5^{k}\right)=\frac{1}{4}\left(15^{k}+9^{k}+(8-i)^{k}+(8+i)^{k}\right), \\
& \theta_{2}\left(5^{k}\right)=\frac{1}{4}\left(15^{k}-9^{k}-i(8-i)^{k}+i(8+i)^{k}\right), \\
& \theta_{3}\left(5^{k}\right)=\frac{1}{4}\left(15^{k}-9^{k}+i(8-i)^{k}-i(8+i)^{k}\right), \\
& \theta_{4}\left(5^{k}\right)=\frac{1}{4}\left(15^{k}+9^{k}-(8-i)^{k}-(8+i)^{k}\right) .
\end{aligned}
$$

From these it follows that

$$
\theta_{0}\left(5^{k}\right)=\frac{1}{2} 5^{k}\left(5^{k}+1\right)-15^{k} .
$$

5. The case $p=2$. In the case $p=2$, Cor. 4 of Theorem 1 reads as follows:

(1) If $n=2^{\alpha_{1}}+\ldots+2^{\alpha_{r}}, \alpha_{1}>\ldots>\alpha_{r}$, then

$$
\theta(n)=\sum_{i=1}^{r} 2^{i-1} \cdot 3^{\alpha_{i}}
$$

Since every $n$ is of one of the three forms:

(i) $2^{\alpha_{1}}+\ldots+2^{\alpha_{r}}$ with $\alpha_{1}>\ldots>\alpha_{r}>0$;

(ii) $2^{\alpha_{1}}+\ldots+2^{\alpha_{r}}+2^{s}+2^{s-1}+\ldots+2+1$ with $\alpha_{1}>\ldots>\alpha_{r}>s+1$;

(iii) $2^{s}+2^{s-1}+\ldots+2+1$

we can use (1) to compute $\theta(n+1)-\theta(n)$ finding its values in the three cases to be $2^{r}, 2^{s+r}, 2^{s+1}$ respectively. Hence we have the result:

(2) the number of odd $\left(\begin{array}{l}n \\ m\end{array}\right)$ for fixed $n$ and $0 \leqslant m \leqslant n$ is equal to $2^{s}$ where $s$ is the number of non-zero digits in the binary expansion of $n$.

This result was proved by Glaisher (2) from our Lemma 1. From this we have the special result, which can be proved in a very nice way directly (3, p. 15 problem 12 and the solution pp. 97-98), that the $n$th row of Pascal's triangle consists of odd numbers exclusively if and only if $n$ is a power of 2. This special case is also an immediate consequence of the corollary to Lemma 3. 
If we let $\theta_{n}=\theta_{1}(n+1)-\theta_{1}(n)$ and $E_{n}=\theta_{0}(n+1)-\theta_{0}(n)$ we have the result:

(3) $E_{n}<\theta_{n}$ if and only if $n+1<2^{1+s}$ where $s$ is the number of non-zero digits in the binary expansion of $n$. In all other cases $E_{n}>\theta_{n}$.

The first statement in (3) follows from (2) since $E_{n}-\theta_{n}=n+1-2^{*}$. In order to prove the second part of (3) suppose the contrary. That is, suppose $E_{n}=\theta_{n}$ for some $n$. Then by $(2), n+1=2^{1+s}$ or $n=2^{s}+\ldots+1$. But then the number of non-zero digits in the binary expansion of $n$ is $s+1$. This is a contradiction and therefore $E_{n} \neq \theta_{n}$ for all $n$.

We include one other result whose proof we omit.

(4) $\theta_{1}(n)>\theta_{0}(n)$ if and only if $1 \leqslant n \leqslant 18$.

6. "Most binomial coefficients are divisible by a given prime". In this section we prove the

Theorem 2.

$$
\lim _{n \rightarrow \infty} \theta(n) / \theta_{0}(n)=0 .
$$

Proof. Clearly $\theta(n)$ and $\theta_{0}(n)$ are non-decreasing functions of $n$. Hence if $p^{k} \leqslant n<p^{k+1}$ then, using Cor. 3(b) of Theorem 1,

$$
\begin{aligned}
& \theta(n) / \theta_{0}(n) \leqslant \theta\left(p^{k+1}\right) / \theta_{0}\left(p^{k}\right) \\
& =\left(\begin{array}{c}
p+1 \\
2
\end{array}\right)^{k+1}\left\{\left(\begin{array}{c}
p^{k}+1 \\
2
\end{array}\right)-\left(\begin{array}{c}
p+1 \\
2
\end{array}\right)^{k}\right\}^{-1}=p(p+1)\left\{\left(\frac{2 p}{p+1}\right)^{k}+\left(\frac{2}{p+1}\right)^{k}-2\right\}^{-1},
\end{aligned}
$$

and this tends to 0 as $n \rightarrow \infty$.

\section{REFERENCES}

1. L. E. Dickson, History of the Theory of Numbers, vol. 1 (Chelsea, New York, 1952).

2. J. W. L. Glaisher, On the residue of a binomial theorem coeffcient with respect to a prime modulus, Quart. J. Math., 30 (1899), 150-156.

3. I. M. Vinogradoff, An Introduction to the Theory of Numbers, (London and New York, Pergamon Press, 1955).

Wesleyan University,

Middletown, Conn.

and

Reed College

Portland, Oregon. 